\title{
Immune Checkpoint Inhibition in Gastro-Oesophageal Cancer
}

\author{
Elizabeth Smytha $^{\text {a }}$ Peter C. Thuss-Patience ${ }^{b}$ \\ ${ }^{a}$ Gastrointestinal Oncology \& Lymphoma Unit, Royal Marsden Hospital, London, UK; \\ ${ }^{\mathrm{b}}$ Medical Clinic for Haematology, Oncology and Tumour-Immunology, Campus Virchow Klinikum, Charité - \\ University Medicine Berlin, Berlin, Germany
}

\section{Keywords}

Checkpoint inhibitor · Chemotherapy · Oesophageal cancer · Gastrointestinal cancer · Immunotherapy

\section{Summary}

Recently some progress has been made in the palliative treatment of gastric cancer. It was shown that secondline chemotherapy and VEGF-R2-directed treatment can prolong survival. Despite these advances most patients with metastatic gastric cancer live for less than 2 years. Immune-checkpoint blockade with anti-CTLA4, anti-PD-1 and anti-PD-L1 antibodies has revolutionised the treatment of many cancers. Significant benefit was also proven in gastric adenocarcinomas. Nivolumab improves overall survival as third-line treatment in Asian gastric cancer patients and is already registered in Japan. Pembrolizumab shows significant efficacy, especially in PD-L1-positive patients as third-line treatment and is FDA approved for this indication. Trials with avelumab are promising and studies with atezolizumab and durvalumab are also on the way. To extend the subgroup of benefitting patients combinations of PD-1/ PD-L1 antibodies with CTLA4, or VEGF-R2 antibodies or combination with chemotherapy are investigated and show promising results. In this article, the existing evidence of PD1 and PD-L1 blockade as monotherapy or in combination with anti-CTLA4, anti-VEGF-R2 and chemotherapy in gastro-oesophageal adenocarcinoma is reviewed and put into perspective.

(c) 2018 S. Karger GmbH, Freiburg

\section{Introduction}

Gastric adenocarcinoma was diagnosed in an estimated 951,600 patients in 2012 worldwide, whereas oesophageal adenocarcinoma adds more than 50,000 cases annually and is rapidly increasing in incidence $[1,2]$. In cases of advanced gastro-oesophageal cancer, the available treatments are limited. Platinum- and fluoropyrimidine-based chemotherapy in the first line setting is generally associated with a survival of less than 1 year in clinical trials $[3,4]$. In the subgroup of HER2-positive gastro-oesophageal cancer targeted treatment with trastuzumab can prolong survival to 16 months [5]. Second-line chemotherapy is proven to prolong survival compared to best supportive care (BSC) $[6,7]$. The addition of the antiVEGFR2 monoclonal antibody ramucirumab to paclitaxel also further improves survival [8]. Despite these advances most patients with metastatic gastro-oesophageal cancer live for less than 2 years. Immune-checkpoint blockade with anti-CTLA4 (cytotoxic T-lymphocyte-associated protein 4), anti-PD-1 (programmed-death receptor 1) and anti-PD-L1 (programmed-death ligand 1) monoclonal antibodies has revolutionised the treatment of melanoma, nonsmall cell lung cancer and bladder cancer. Therefore, assessment of the efficacy of modern immunotherapies has been eagerly awaited by patients and investigators treating gastro-oesophageal cancer. The aim of this review is to explore the recently published and presented results of trials of immune-checkpoint blockade in gastrooesophageal adenocarcinoma and to reflect on the molecular qualities which might predict for response to immunological intervention. Selected trials of checkpoint inhibition are shown in table 1.

\section{KARGER}

() 2018 S. Karger GmbH, Freiburg
PD Dr. med. Peter Thuss-Patience 


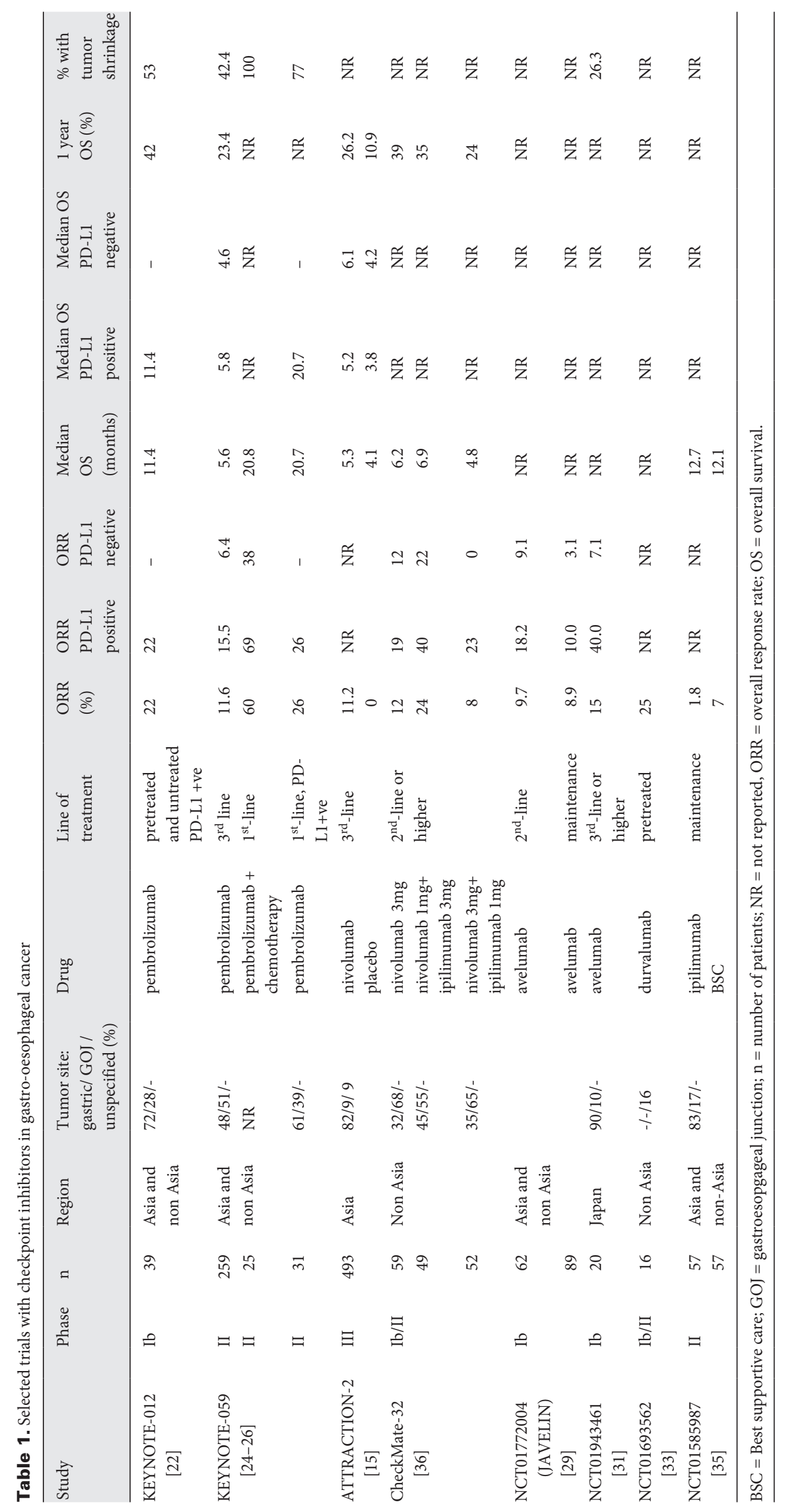




\section{Anti-PD-1 Therapy Gastro-Oesophageal Adenocarcinoma}

\section{Nivolumab in ATTRACTION-02}

The anti-PD-1 monoclonal antibody nivolumab is a humanised immunoglobulin G4 antibody which is licensed to treat multiple cancers including melanoma, non-small cell lung cancer and renal cell carcinoma, bladder cancer, cancer of the head and neck and Hodgkin's disease [9-14]. On $23^{\text {rd }}$ of September 2017 nivolumab was licensed to treat chemo-refractory gastro-oesophageal cancer in Japan, based on the results of the ATTRACTION-02 trial. In this phase-III, randomised study patients with advanced or recurrent gastro-oesophageal cancer, who had progressed on or who were intolerant of $\geq 2$ standard chemotherapy regimens, were treated with nivolumab $3 \mathrm{mg} / \mathrm{kg}$ every 2 weeks or placebo [15]. The trial recruited only patients from 3 Asian countries (Japan, Korea and Taiwan). ATTRACTION-02 was a large study; 493 patients were recruited and were randomised in a 2:1 ratio using stratification factors of country of origin, ECOG PS and number of metastases. Patients treated with nivolumab displayed significantly improved median overall survival (OS); this increased from 4.14 months with placebo to 5.32 months with nivolumab (HR 0.63; 95\% CI (0.50$0.78), \mathrm{p}<0.0001)$. Importantly, survival at 1 and 2 years more than doubled for patients who were treated with nivolumab; at twelve months this was $26.6 \%$ and $10.9 \%$ in nivolumab- and placebotreated patients respectively. Radiological response rates were relatively modest with nivolumab, some degree of tumour shrinkage was observed in up to $40 \%$ of nivolumab-treated patients, Response Evaluation Criteria in Solid Tumours (RECIST) were achieved in $12 \%$ of patients with a median duration of response of 9.5 months. In ATTRACTION-02, PD-L1 status was assessed retrospectively, and is, therefore, only available for a limited number of patients (39\% of the cohort). PD-L1 was assessed only on tumour cells using the 28-8 pharmDx assay. In ATTRACTION-02, PD-L1 positivity as defined by the presence of PD-L1 staining $\geq 1 \%$ of tumour cells was demonstrated in 26/192 (13.5\%) of patients. With the caveat of the limited number of cases assessed for PD-L1 status, there did not appear to be a significant differential outcome in terms of nivolumab efficacy in PD-L1-positive and PD-L1-negative patients. Therefore, nivolumab was licensed in Japan in for a biomarker unselected, chemo-refractory gastro-oesophageal cancer population.

Considering the toxicities associated with cytotoxic chemotherapy, treatment with nivolumab in patients with chemo-refractory gastro-oesophageal cancer was well tolerated. The proportion of patients who had a toxicity which led to discontinuation of treatment was very low $(1 \%, n=4)$. No new safety concerns were demonstrated in this distinct patient population.

\section{Nivolumab in CheckMate-032}

One reasonable question is whether the data from the ATTRACTION-2 trial, which recruited only Asian patients, can be generalised to a global population. This is relevant because patients with gastrooesophageal cancer in Asia have, stage for stage, better survival than patients from non-Asian countries and may have a different immune-infiltrate compared with non-Asian patients [16]. Nivolumab has also been evaluated in non-Asian patients in the CheckMate-032 trial which was a non-randomized Phase I/II cohort-study of nivolumab monotherapy and combination nivolumab + ipilimumab (anti-CTLA4) in non-Asian, PD-L1-unselected gastro-oesophageal cancer patients. In the single agent nivolumab cohort of CheckMate-032 59 patients were treated with nivolumab $3 \mathrm{mg} / \mathrm{kg}$ once every 2 weeks. There were similarities between this population and that of ATTRACTION-02; almost half of patients were treated with at least 3 prior chemotherapy regimens $(n=29,49 \%)$. In a higher percentage, the tumour was located in the gastro-oesophageal junction (68\%) compared to the ATTRACTION-02 population (9\%). Strikingly similarly to ATTRACTION-02, the radiological response rate observed in nivolumab-treated patients was $12 \%(\mathrm{n}=7)$. PD-L1 status was available for 42 patients, of these $38 \%$ were $\mathrm{PD}-\mathrm{L} 1$ positive (PD-L1 staining in $>1 \%$ tumour cells). Although the overall response rate (ORR) was marginally higher in PD-L1-positive patients, responses were observed in PD-L1-positive and -negative tumours (12\% PD-L1 negative vs. $19 \%$ PD-L1 positive). In CheckMate-032 median OS for nivolumab treated patients regardless of biomarker status was 6.2 months (95\% CI 3.4-12.4 months), again similar to ATTRACTION-02. No new safety signals were observed in the non-Asian population treated with nivolumab in CheckMate-032, suggesting anti-PD-1 therapy was equally well tolerated in both Asian and non-Asian populations.

In view of the similarity in radiological response rates and survival observed in the ATTRACTION-02 and CheckMate-032 trials, it would be reasonable to suggest that the survival benefit demonstrated in the randomised ATTRACTION-02 trial might be generalisable to a non-Asian chemo-refractory gastro-oesophageal cancer population.

\section{Anti-PD-1 Therapy Using Pembrolizumab: KEYNOTE-012 and KEYNOTE-059}

A second monoclonal PD-1 antibody, pembrolizumab, has taken a different pathway to regulatory approval in the United States. Pembrolizumab is a humanised immunoglobulin G4 monoclonal antibody which has approvals in melanoma, non-small cell lung cancer, Hodgkin's disease, bladder cancer and microsatelliteunstable cancers [17-21]. The first published data relating to efficacy of anti-PD-1 therapy in gastro-oesophageal cancer were the KEYNOTE-012 study of pembrolizumab in PD-L1-selected previously treated gastro-oesophageal cancer. Unlike the studies with nivolumab, in the KEYNOTE-12 patients were selected for PD-L1positive status; however, a different antibody (QualTek 22C3) and scoring system were used. Eligibility was considered if patients had $\geq 1 \%$-positive PD-L1 staining on tumour cells or contiguous immune infiltrate. Approximately $40 \%$ of screened patients were pos- 
itive and 39 of these were enrolled in the phase-Ib trial. In this heavily pre-treated cohort, the radiological response rate for patients with evaluable disease $(n=36)$ was $22 \%$ and was comparable for Asian and non-Asian patients [22]. Interestingly, patients treated on KEYNOTE-012 were required to undergo a biopsy prior to starting treatment on study, and when pre-treatment study and on-study biopsies were compared, $25 \%$ of patients previously $\mathrm{PD}-\mathrm{L} 1$ positive were now $\mathrm{PD}-\mathrm{L} 1$ negative. This may represent the dynamic status of PD-L1 expression, biomarker heterogeneity or differences in the assay used, and demonstrates the lack of reliability of PD-L1 as a biomarker [23].

Following the encouraging results of the initial KEYNOTE-012 study, pembrolizumab was further evaluated in the large, multicohort KEYNOTE-059 study. This trial variously evaluated pembrolizumab as a single agent in patients with treatment-refractory gastro-oesophageal cancer unselected for PD-L1 status (KEYNOTE-059 cohort 1), in PD-L1-positive treatment naïve gastrooesophageal cancers (KEYNOTE-059 cohort 3 ) and in conjunction with chemotherapy in patients with untreated PD-L1-unselected gastro-oesophageal cancer (KEYNOTE-059 cohort 2) [24-26].

The results of KEYNOTE-059 (cohort 1) lead to the registration of pembrolizumab for chemo-refractory gastro-oesophageal cancer which is PD-L1 positive. In this open label non-randomised trial, 259 patients with gastro-oesophageal cancer, who were refractory at least 2 lines of chemotherapy, received treatment with pembrolizumab $200 \mathrm{mg}$ every 3 weeks. As this was a predominantly nonAsian trial, tumours of the gastro-oesophageal cancer were enriched in the population (51\%). All patients were assessed prospectively for PD-L1 status; a specific scoring system was used. This is described as the combined positive score (CPS) where CPS is calculated as the absolute number PD-L1-positive cells of any type (tumour or immune) divided by total number of tumour cells $\mathrm{x} 100$ ). Using the CPS score and the 22C3 antibody, 148/259 (57\%) of patients in KEYNOTE-059 cohort 1 were considered to be $\mathrm{PD}-\mathrm{L} 1$ positive.

In keeping with what has been observed for nivolumab in the ATTRACTION-02 study, in KEYNOTE-059 radiological responses were seen in $12 \%$ of unselected patients and $42 \%$ of patients experienced some tumour shrinkage. There was a differential in response between patients who were PD-L1 positive and those who were PD-L1 negative; response rates were $15.5 \%$ vs. $6.4 \%$ respectively. In patients who achieved a radiological response, the duration of response was 8.4 months for PD-L1-unselected patients, 16.3 months for PD-L1-positive patients and 6.9 months for PD-L1 negative. Thus, although the US approval for pembrolizumab is for PD-L1positive tumours only, responses and a reasonable duration of response were observed also in PD-L1-negative patients.

In KEYNOTE-059 cohort 1 there was a suggestion that checkpoint-immune blockade might be more helpful in earlier lines of therapy. Radiological responses were more common in patients who received treatment after 2 lines of chemotherapy (16.4\%) rather than 3 (6.4\%).

The median progression free survival (PFS) for pembrolizumab in KEYNOTE-059 cohort 1 was 2 months (95\% CI 2.0-2.1 months), which would not appear to be superior to chemotherapy in this set- ting, although this is perhaps not the best metric by which to evaluate the efficacy of immunotherapy. Only a small subset of patients seem to benefit, but those who benefit do so for a long time. Similarly, the median OS in KEYNOTE-059 cohort 1 was 5.5 months (4.2-6.5 months) for unselected patients, which is almost identical to that observed with nivolumab in ATTRACTION-02. There was no difference in PFS according to PD-L1 status (2.1 vs. 2.0 months respectively). Patients who were $\mathrm{PD}-\mathrm{L} 1$ positive appeared to have marginally improved median OS compared to PD-L1 negative (5.8 months vs. 4.6 months), and 6 month OS of $48.4 \%$ vs. $42.9 \%$. A group of special interest is patients with microsatellite instabilityhigh (MSI-H) tumours. In KEYNOTE-059 these made up 4\% of the population $(\mathrm{n}=7)$. As expected, radiological response rates, seen in 4 of 7 patients, were high (57\%), emphasising the importance of considering all patients with advanced cancer for assessment of mismatch-repair deficiency in their tumours.

In view of the encouraging response rates demonstrated in treatment refractory patients and the possibility of increased benefit in early lines of therapy, pembrolizumab was also assessed in a cohort of PD-L1-positive-treatment naïve patients in the KEYNOTE-059 study (cohort 3). The preliminary results from this cohort have been presented $[25,27]$. The 31 patients with untreated, PD-L1-positive gastro-oesophageal adenocarcinoma were treated with pembrolizumab $200 \mathrm{mg}$ once every 3 weeks. The radiological response rate reported was $26 \%$ (95\% CI $12-45 \%)$; of these $7 \%$ had a complete radiological response and $77 \%$ had some evidence of tumour shrinkage. Although encouraging, and superior to the response rate observed in subsequent lines of therapy, this is not equivalent to cytotoxic chemotherapy in this setting where response rates of more $40 \%$ are expected. However, at the time of reporting, with limited follow-up, median OS for this group was 20.7 months (95\% CI 9.2-20.7 months) which is superior to that observed with chemotherapy. Further follow-up will be required to validate these findings, and single-agent pembrolizumab is currently under evaluation as 1 of the treatment arms in the first-line randomised KEYNOTE-062 trial [28].

At the time of writing, the results of the KEYNOTE-061 trial have been reported in short form only as a press release. In this trial, patients with previously treated gastro-oesophageal cancer, who had received 1 line of therapy, were treated with pembrolizumab or paclitaxel chemotherapy. The primary endpoint of the trial, an improvement of median OS in PD-L1-positive patients (HR, 0.82 [95\% CI, 0.66-1.03]; $\mathrm{p}=0.042$ [one-sided]) was not met. These results are disappointing, in particular in view of the PD-L1selected nature of the patients.

\section{Targeting PD-L1 in Gastro-Oesophageal Cancer with Avelumab}

Avelumab is a fully humanised anti-PD-L1 antibody which is licensed for the treatment of Merkel cell carcinoma. Avelumab can induce ADCC (antibody-derived cellular cytotoxicity) due to the native Fc-region contained. Avelumab has been assessed as a sec- 
ond line treatment and as a maintenance therapy in gastro-oesophageal cancer. All of the 89 patients were treated with maintenance (following chemotherapy) avelumab $10 \mathrm{mg}$ once every 2 weeks in the JAVELIN phase-Ib study [29]. Treatment in the maintenance setting had a median duration of 12 weeks (range 2-62 weeks) which is somewhat equivalent to PFS on second line chemotherapy for gastro-oesophageal cancer. Patients were tested for PD-L1 using Dako Clone $73-10$; of these $38.5 \%$ were PD-L1 positive at the $1 \%$ level on tumour cells. Patients treated with maintenance avelumab had a radiological response rate of $9.0 \%$ (5/55) There was a suggestion that radiological response rates were higher in patients who expressed PD-L1 (10\% vs. 3\% for PD-L1 positive vs. negative), albeit in small numbers of patients $(n=52)$. Progression free survival was also slightly longer for PD-L1 positive avelumab treated patients (17.6 vs. 11.6 weeks for PD-L1 positive vs. negative). Results of a randomised phase-III trial investigating the effect of Avelumabmaintenance treatment are awaited (JAVELIN 100 Gastric)[30].

In an initial report of patients treated with avelumab at later lines of therapy, radiological response rate was like that demonstrated for maintenance avelumab in that 6 out of 62 treated patients had a radiological response (9.7\%)[29]. Again, although a small number of patients had material available upon which to perform PD-L1 testing, it appeared that radiological responses were more common in patients who had PD-L1-positive tumours ( $18.2 \%$ vs. $9.1 \%$ for $1 \%$ expression level). This was more pronounced in patients who had expression of PD-L1 in at least $5 \%$ of cells $(28.6 \%$ vs. $6.7 \%)$. In a small phase-Ib trial in Japanese patients (JAVELIN solid Tumor JPN; NCT01943461) 20 PD-L1-unselected patients with at least 2 prior treatment lines were treated with avelumab. A radiologic response was achieved in $15 \%$ of patients with a PFS of 12.3 weeks [31]. Therefore, initial results suggested PD-L1 inhibition appears to be of similar activity to PD-1 inhibition in gastro-oesophageal cancer.

However, at the time of writing, preliminary results of the Javelin 300 trial have been released which showed no OS benefit for third-line avelumab compared to investigators choice of chemotherapy (irinotecan or taxanes)[32]. However, in this study, the bar was set much higher than in the ATTRACTION-02 study where the comparator arm was placebo and full results still need to be seen.

\section{Targeting PD-L1 with Durvalumab}

The PD-L1 antibody durvalumab was investigated in a phase$\mathrm{Ib} / \mathrm{II}$-multicenter trial for advanced solid tumours in 346 patients. In a cohort of 16 gastro-oesophageal tumours, 3 partial remissions and 1 complete response could be detected [33]. Currently durvalumab is investigated in gastro-oesophageal tumours in several trials as monotherapy, or in combination with CTLA-4 blockade, chemotherapy, or chemoradiotherapy.

\section{Targeting PD-L1 with Atezolizumab}

Only very limited data for atezolizumab in gastro-oesophageal cancer are available. The PD-L1 antibody atezolizumab has been investigated in a phase-I trial for multiple tumour types in $171 \mathrm{pa}$ tients. Only 1 patient with gastro-oesophageal cancer was included. This patient showed a partial remission [34].

\section{Targeting CTLA4 with Ipilimumab}

The anti-CTLA4 antibody ipilimumab was evaluated in a randomised trial as a switch-maintenance therapy in patients who were benefiting from (stable disease or better), from first line chemotherapy [35]. All of the 57 patients were randomised to ipilimumab $10 \mathrm{mg} / \mathrm{kg}$ every 3 weeks or to BSC. The primary endpoint of the trial was not met as immune-related PFS for ipilimumab was 2.92 compared to 4.90 months for BSC. (HR $=1.44$; (80\% CI, 1.09$1.91 ; \mathrm{p}=0.097$ ) leading to closure at an interim analysis. Therefore, ipilimumab does not provide sufficient benefit in gastro-oesophageal cancer to develop as a single agent, but may be considered in combinations as discussed below.

\section{Combination Therapy}

Combination Immunotherapy with Anti-PD-1 plus Anti-CTLA4 Antibodies: CheckMate-032

As many of the patients with gastro-oesophageal cancer are PD-L1 negative, combination-immune-checkpoint blockade is attractive in this disease. Furthermore, even gastro-oesophageal cancer patients who are PD-L1 positive have quite limited benefit from anti-PD-1 therapy alone, therefore increasing the benefit due to checkpoint-immune blockade through use of combinations may be helpful.

The CheckMate-032 study assessed the benefit of nivolumab single-agent treatment in 1 cohort and the 2 other cohorts evaluated 2 dose levels of nivolumab plus ipilimumab-combination therapy. The dose levels in these 2 arms were nivolumab $3 \mathrm{mg} / \mathrm{kg}$ plus ipilimumab $1 \mathrm{mg} / \mathrm{kg}$ every 3 weeks (N3:I1) and nivolumab $1 \mathrm{mg} / \mathrm{kg}$ plus ipilimumab $3 \mathrm{mg} / \mathrm{kg}$ every 3 weeks (N1:13) [36]. Comparing the 2 combination arms of the trial patients treated with N1:I3 had higher radiological response rates (24\% vs. $8 \%$ ) which also translated into higher survival at 1 year (35\% vs. $24 \%$ ) and at 18 months (28\% vs. $13 \%$ for N1:I3, N3:I1 respectively) The regimen chosen for further evaluation in CheckMate-649 is the nivolumab $1 \mathrm{mg}$ / ipilimumab $3 \mathrm{mg} /$ dosing schedule. $\mathrm{PD}-\mathrm{L} 1$ positivity predicted for increased benefit from combination therapy, up to $40 \%$ of patients experienced an objective response in the N1:I3 arm.

Combination-immune-checkpoint blockade is associated with increased toxicity compared to single-agent anti-PD-1 or anti-PDL1 therapy. Patients treated with higher dose ipilimumab had significant rates of grade 3 and 4 adverse events (35\%), which was twice the rate observed with lower dose ipilimumab (17\%) and greater than single-agent nivolumab (5\%). 


\section{Combination Anti-PD-1 Plus Chemotherapy: KEYNOTE-059 \\ Cohort 2}

In view of the limited response rates observed with single-agent anti-PD-1 therapy, combination with chemotherapy to effect tumour shrinkage in addition to activating the immune system is of interest. In a small cohort of KEYNOTE-059 (cohort 2), pembrolizumab plus cisplatin-fluoropyrimidine chemotherapy was used in patients who were PD-L1-unselected and had treatment-naive gastro-oesophageal cancer which was HER2-negative [37]. The trial was run globally and 25 patients of Asian and non-Asian origin were treated, of whom $64 \%$ were PD-L1-positive using the previously described CPS score. Combination therapy with pembrolizumab and chemotherapy was associated with an encouraging radiological response rate of 60\% (95\% CI 39\%-69\%) in all patients, regardless of biomarker status. $\mathrm{PD}$-L1-positive patients appeared to derive more benefit from the combination than PD-L1-negative patients (ORR 69\% vs. 38\% respectively). With the caveat of limited follow-up and small numbers, median OS was promising; this was 13.8 months for the entire cohort. The toxicities observed in the trial did not appear to be immune-related. Several combinations of chemotherapy and anti-PD-1 and anti-PD-L1 monoclonal antibodies are currently being evaluated in randomised controlled trials.

\section{Combination Anti-PD-1 and Ramucirumab: JVDF Trial and NCT 02572687}

As the anti-VEGFR2, monoclonal antibody ramucirumab is licensed for the treatment of previously-treated gastro-oesophageal cancer, there is also interest in combining this therapy with immune-checkpoint blockade. Furthermore, there is a preclinical rationale to combine anti-angiogenic therapy with immunotherapy; this is because VEGF signalling appears to counter the natural response of the immune system $[38,39]$. There were 2 cohorts in the JVDF trial, untreated and previously-treated patients. In patients who had previously been treated with at least 1 line of chemotherapy $(\mathrm{n}=41)$, the ORR to ramucirumab plus pembrolizumab was $7 \%$. The disease control rate (complete responses + partial responses + stable disease) was $51 \%$, which is somewhat higher than seen for single-agent pembrolizumab in KEYNOTE-059 (27.8\%). The 12 -month OS rate was $34.6 \%$, which is quite promising. Median PFS was 2.6 months (95\% CI 1.5-4.2 months) and median OS was 6.4 months (95\% 4.2-12.6 months) respectively, not much different from single-agent pembrolizumab in KEYNOTE-059, but the 12 -month OS rate of $34.6 \%$ (compared to $23 \%$ in KEYNOTE-059) may point to some additional benefit of the combination with ramucirumab and makes VEGF-R blockade a very interesting partner for checkpoint inhibition. Results were also encouraging in patients treated with the combination pembrolizumab plus ramucirumab in the first line setting. In 28 treatment naïve patients with advanced gastro-oesophageal cancer, unselected for PD-L1 (PD-L1 positivity in 68\%) the combination of ramucirumab and pembrolizumab resulted in $77 \%$ of patients in a decrease of tumour size (including 4 PD-L1-negative patients) an ORR of 25\% and a disease control of $68 \%$ with a median duration of response of 10 months [40].

The toxicity of ramucirumab plus pembrolizumab corresponded to both single agents without any additive toxicity.

Durvalumab and ramucirumab were also combined in 29 pretreated PD-L1 unselected (48\% PD-L1 positive) (NCT 02572687) gastro-oesophageal cancer patients and reached a 55\% DCR (17\% ORR) with $38 \%$ of patients experiencing a tumour shrinkage [41].

\section{Gastro-Oesophageal Cancer Biology and Immunotherapy}

\section{Gastric-Cancer Subtypes and Immune Response}

Gastric cancer has recently been molecularly characterised by The Cancer Genome Atlas (TCGA) group using a multi-platform approach which described 4 gastric adenocarcinoma subtypes: these were the chromosomally unstable (CIN, 50\%), genomically stable, (GS, 20\%), microsatellite unstable (MSI, 21\%), and Epstein-Barr Virus (EBV, 9\%) subtypes [42]. These subtypes demonstrate diversity in immunological targets, with EBV and MSI subtypes the most promising in this regard. Amplification of 9p encoding PD-L1 and PD-L2 genes is often found in EBV-positive tumours, and high levels of $\mathrm{PD}-\mathrm{L} 1$ protein expression has been demonstrated on tumour cells in EBV-positive gastric adenocarcinoma [43]. Germline or acquired defects in mismatch repair processes lead to high levels of mutations and neo-antigen presentation in MSI gastric cancer, which in turn leads to tumour-infiltrating lymphocyte invasion and PD-L1 expression $[44,45]$. Both EBV and MSI gastric adenocarcinoma have high levels expression of gene signatures which are associated with response to PD-1 therapy [22, 43]. However, although EBV and MSI gastric cancer may be more likely to benefit from immunotherapy, because of their innate positive prognostic value, these subtypes are less often found in patients with advanced disease than other subtypes. For example, MSI-H tumours made up $4 \%$ of the KEYNOTE-059 cohort. Conversely, CIN gastric cancer, which extends to include oesophageal adenocarcinoma, appears to have molecular characteristics which make it less likely to respond to checkpoint inhibitor therapy. The IFN- $\gamma$ response gene signature is less frequently present in CIN gastric cancer, and cytokines, for example INF- $\gamma$, IL1B, IL-2, IL-3, IL-21 and IL-27 and which are commonly expressed at high levels in EBV gastric cancer may be expressed at lower levels in CIN cancers [46]. In other cancers such as melanoma, copy number alterations which are observed in aneuploid tumours are associated with low levels of immune infiltrate and immune evasion; this phenomenon is also likely to affect gastric and gastro-oesophageal adenocarcinoma of CIN subtype [47]. Finally, within CIN gastric and gastro-oesophageal cancers, whole exome sequencing revealed a population of tumours which are microsatellite stable, but which have an increased mutation burden relative to other gastro-oesophageal tumours [48]. This could be a subset of CIN cancers potentially sensitive to immune checkpoint inhibition. 


\section{Conclusions and Future Directions}

Single-agent-PD-1 blockade in gastro-oesophageal cancer results in response rates of approximately $10-20 \%$ depending on the number of previous lines of chemotherapy and PD-L1 status. In general, this appears to be a class effect and does not differ between antibodies. Nivolumab was well tolerated, statistically significantly improved OS, and more than doubles 1-year survival compared to placebo in chemo-refractory gastro-oesophageal cancer in the ATTRACTION-2 trial. Therefore, there is level 1 evidence to support use of nivolumab in PD-L1-unselected gastro-oesophageal Asian cancer patients who have been previously treated with $\geq 2$ lines of chemotherapy. Although pembrolizumab has not been evaluated in a randomised trial, the radiological response rates and survival attributed to pembrolizumab in KEYNOTE-059 appear to be almost identical to those observed in ATTRACTION-02. The key difference between the studies is that PD-L1 appeared to be a better discriminator of benefit for patients treated with pembrolizumab in KEYNOTE-059 than for nivolumab in ATTRACTION-02. However, due to lack of standardisation in assays and methodology of assessment, PD-L1 can be considered at best an enrichment biomarker for response, and is insufficient to negatively select patients for anti-PD-1 therapy. The PD-L1 antibody avelumab demonstrated activity in a similar range to PD-1 antibodies and although the second line trial was negative, results of the maintenancephase-III trials is awaited. More data for atezolizumab and durvalumab in gastro-oesophageal cancer need to be seen. Combination of anti-PD-1 and anti-CTLA4 therapy is associated with improvements in objective response rates when compared to singleagent anti-PD-1 therapy, however, also with increased rates of toxicity. Larger trials are required to understand whether combination-immune-checkpoint blockade will result in improved OS for patients with gastro-oesophageal cancer. Combinations of antiPD-1 therapy, chemotherapy, or VEGFR-2 antibodies are of sig- nificant interest, however, these combinations have been evaluated in small numbers of patients, and the results of ongoing trials are awaited.

Two recent reports have brought the disappointing news of the failure of pembrolizumab and avelumab when compared to chemotherapy in previously-treated patients with gastro-oesophageal cancer. Therefore, more work on biomarkers is needed to allow selection of the patients who are most likely to benefit from singleagent-checkpoint blockade. Combination therapies to increase the proportion of patients who benefit will be necessary to fully harness the power of novel immunotherapies for patients with gastrooesophageal cancer. Future combinations of interest include doublet immunotherapy for example nivolumab plus anti-LAG3 antibodies, combinations with targeted therapy such as andecaliximab, poly ADP ribose polymerase (PARP) inhibitors or inhibitors of the Wnt pathway.

For the moment, because checkpoint inhibitors are currently not licensed in Europe, the authors do not recommend its use outside of a clinical trial. We foresee that checkpoint inhibition will have its place in third line treatment in the near future. On an individual patient basis MSI-H tumours may already be considered for treatment with checkpoint inhibitors after failure of chemotherapy. To facilitate further development of immune-checkpoint inhibition, patient and oncologist engagement with clinical trials is a prerequisite.

\section{Disclosure Statement}

Dr Smyth acknowledges consulting fees for an advisory role from Five Prime Therapeutics, Servier, Gritstone Oncology, BMS and Celgene and funding support of the National Institute for Health Research Royal Marsden Institute for Cancer Research Biomedical Research Centre. Dr Thuss-Patience acknowledges consulting fees for an advisory role from MSD, BMS, Merck, Roche, Lilly, Nordic and Pfizer.

\section{References}

1 Torre LA, Bray F, Siegel RL, Ferlay J, Lortet-Tieulent J, Jemal A: Global cancer statistics, 2012. CA Cancer J Clin 2015;65:87-108.

2 Edgren G, Adami HO, Weiderpass E, Nyren O: A global assessment of the oesophageal adenocarcinoma epidemic. Gut 2013;62:1406-1414.

3 Cunningham D, Starling N, Rao S, Iveson T, Nicolson M, Coxon F, Middleton G, Daniel F, Oates J, Norman AR: Capecitabine and oxaliplatin for advanced esophagogastric cancer. N Engl J Med 2008;358:36-46.

4 Van Cutsem E, Moiseyenko VM, Tjulandin S, Majlis A, Constenla M, Boni C, Rodrigues A, Fodor M, Chao Y, Voznyi E, Risse M-L, Ajani JA: Phase III Study of Docetaxel and Cisplatin plus fluorouracil compared with cisplatin and fluorouracil as first-line therapy for advanced gastric cancer: a report of the V325 study group. J Clin Oncol 2006;24:4991-4997.
5 Bang Y-J, Van Cutsem E, Feyereislova A, Chung HC, Shen L, Sawaki A, Lordick F, Ohtsu A, Omuro Y, Satoh T, Aprile G, Kulikov E, Hill J, Lehle M, Rüschoff J, Kang Y-K: Trastuzumab in combination with chemotherapy versus chemotherapy alone for treatment of HER2-positive advanced gastric or gastro-oesophageal junction cancer (ToGA): a phase 3, open-label, randomised controlled trial. The Lancet 2010;376:687-697.

6 Thuss-Patience PC, Kretzschmar A, Bichev D, Deist T, Hinke A, Breithaupt K, Dogan Y, Gebauer B, Schumacher G, Reichardt P: Survival advantage for irinotecan versus best supportive care as second-line chemotherapy in gastric cancer - a randomised phase III study of the Arbeitsgemeinschaft Internistische Onkologie (AIO). Eur J Cancer 2011;47:2306-2314.

7 Janowitz T, Thuss-Patience P, Marshall A, Kang JH, Connell C, Cook N, Dunn J, Park SH, Ford H: Chemotherapy vs supportive care alone for relapsed gastric, gastroesophageal junction, and oesophageal adenocarcinoma: a meta-analysis of patient-level data. Br J Cancer 2016;114:381-387.
8 Wilke H, Muro K, Van Cutsem E, Oh SC, Bodoky G, Shimada Y, Hironaka S, Sugimoto N, Lipatov O, Kim TY, Cunningham D, Rougier P, Komatsu Y, Ajani J, Emig M, Carlesi R, Ferry D, Chandrawansa K, Schwartz JD, Ohtsu A: Ramucirumab plus paclitaxel versus placebo plus paclitaxel in patients with previously treated advanced gastric or gastro-oesophageal junction adenocarcinoma (RAINBOW): a double-blind, randomised phase 3 trial. Lancet Oncol 2014;15:1224-1235.

9 Borghaei H, Paz-Ares L, Horn L, Spigel DR, Steins M, Ready NE, Chow LQ, Vokes EE, Felip E, Holgado E, Barlesi F, Kohlhaufl M, Arrieta O, Burgio MA, Fayette J, Lena H, Poddubskaya E, Gerber DE, Gettinger SN, Rudin CM, Rizvi N, Crino L, Blumenschein GR, Jr., Antonia SJ, Dorange C, Harbison CT, Graf Finckenstein F, Brahmer JR: Nivolumab versus docetaxel in advanced nonsquamous non-small-cell lung cancer. N Engl J Med 2015;373:1627-1639. 
10 Larkin J, Chiarion-Sileni V, Gonzalez R, Grob JJ, Cowey CL, Lao CD, Schadendorf D, Dummer R, Smylie M, Rutkowski P, Ferrucci PF, Hill A, Wagstaff J, Carlino MS, Haanen JB, Maio M, Marquez-Rodas I, McArthur GA, Ascierto PA, Long GV, Callahan MK, Postow MA, Grossmann K, Sznol M, Dreno B, Bastholt L, Yang A, Rollin LM, Horak C, Hodi FS, Wolchok JD: Combined nivolumab and ipilimumab or monotherapy in untreated melanoma. N Engl J Med 2015;373:23-34.

11 Motzer RJ, Escudier B, McDermott DF, George S, Hammers HJ, Srinivas S, Tykodi SS, Sosman JA, Procopio G, Plimack ER, Castellano D, Choueiri TK, Gurney H, Donskov F, Bono P, Wagstaff J, Gauler TC, Ueda T, Tomita Y, Schutz FA, Kollmannsberger C, Larkin J, Ravaud A, Simon JS, Xu LA, Waxman IM, Sharma P: Nivolumab versus everolimus in advanced renal-cell carcinoma. N Engl J Med 2015;373:18031813.

12 Sharma P, Callahan MK, Bono P, Kim J, Spiliopoulou P, Calvo E, Pillai RN, Ott PA, de Braud F, Morse M, Le DT, Jaeger D, Chan E, Harbison C, Lin CS, Tschaika M, Azrilevich A, Rosenberg JE: Nivolumab monotherapy in recurrent metastatic urothelial carcinoma (CheckMate 032): a multicentre, open-label, two-stage, multi-arm, phase 1/2 trial. Lancet Oncol 2016;17: 1590-1598.

13 Ferris RL, Blumenschein GJ, Fayette J, Guigay J, Colevas $\mathrm{AD}$, Licitra L, Harrington $\mathrm{K}$, Kasper S, Vokes EE, Even C, Worden F, Saba NF, Iglesias Docampo LC, Haddad R, Rordorf T, Kiyota N, Tahara M, Monga M, Lynch M, Geese WJ, Kopit J, Shaw JW, Gillison ML: Nivolumab for recurrent squamous-cell carcinoma of the head and neck. N Engl J Med 2016;375:1856-1867.

14 Ansell SM, Lesokhin AM, Borrello I, Halwani A, Scott EC, Gutierrez M, Schuster SJ, Millenson MM, Cattry D, Freeman GJ, Rodig SJ, Chapuy B, Ligon AH, Zhu L, Grosso JF, Kim SY, Timmerman JM, Shipp MA, Armand P: PD-1 blockade with nivolumab in relapsed or refractory Hodgkin's lymphoma. N Engl J Med 2015; 372:311-319.

15 Kang YK, Boku N, Satoh T, Ryu MH, Chao Y, Kato K, Chung HC, Chen JS, Muro K, Kang WK, Yeh KH, Yoshikawa T, Oh SC, Bai LY, Tamura T, Lee KW, Hamamoto Y, Kim JG, Chin K, Oh DY, Minashi K, Cho JY, Tsuda M, Chen LT: Nivolumab in patients with advanced gastric or gastro-oesophageal junction cancer refractory to, or intolerant of, at least two previous chemotherapy regimens (ONO-4538-12, ATTRACTION-2): a randomised, double-blind, placebo-controlled, phase 3 trial. Lancet 2017;390:2461-2471.

16 Lin SJ, Gagnon-Bartsch JA, Tan IB, Earle S, Ruff L, Pettinger K, Ylstra B, van Grieken N, Rha SY, Chung HC, Lee J-S, Cheong JH, Noh SH, Aoyama T, Miyagi Y, Tsuburaya A, Yoshikawa T, Ajani JA, Boussioutas A, Yeoh KG, Yong WP, So J, Lee J, Kang WK, Kim S, Kameda Y, Arai T, zur Hausen A, Speed TP, Grabsch HI, Tan P: Signatures of tumour immunity distinguish Asian and non-Asian gastric adenocarcinomas. Gut 2014;64:1721-1731.

17 Robert C, Schachter J, Long GV, Arance A, Grob JJ, Mortier L, Daud A, Carlino MS, McNeil C, Lotem M, Larkin J, Lorigan P, Neyns B, Blank CU, Hamid O, Mateus C, Shapira-Frommer R, Kosh M, Zhou H, Ibrahim N, Ebbinghaus S, Ribas A: Pembrolizumab versus ipilimumab in advanced melanoma. $\mathrm{N}$ Engl J Med 2015;372:2521-2532.

18 Reck M, Rodriguez-Abreu D, Robinson AG, Hui R, Csoszi T, Fulop A, Gottfried M, Peled N, Tafreshi A, Cuffe S, O’Brien M, Rao S, Hotta K, Leiby MA, Lubiniecki GM, Shentu Y, Rangwala R, Brahmer JR: Pembrolizumab versus chemotherapy for PD-L1-positive non-small-cell lung cancer. N Engl J Med 2016; 375:1823-1833.
19 Le DT, Uram JN, Wang H, Bartlett BR, Kemberling H, Eyring AD, Skora AD, Luber BS, Azad NS, Laheru D, Biedrzycki B, Donehower RC, Zaheer A, Fisher GA, Crocenzi TS, Lee JJ, Duffy SM, Goldberg RM, de la Chapelle A, Koshiji M, Bhaijee F, Huebner T, Hruban RH, Wood LD, Cuka N, Pardoll DM, Papadopoulos N, Kinzler KW, Zhou S, Cornish TC, Taube JM, Anders RA, Eshleman JR, Vogelstein B, Diaz LA, Jr.: PD-1 blockade in tumors with mismatch-repair deficiency. N Engl J Med 2015;372:2509-2520.

20 Chen R, Zinzani PL, Fanale MA, Armand P, Johnson NA, Brice P, Radford J, Ribrag V, Molin D, Vassilakopoulos TP, Tomita A, von Tresckow B, Shipp MA, Zhang Y, Ricart AD, Balakumaran A, Moskowitz CH: Phase II study of the efficacy and safety of pembrolizumab for relapsed/refractory classic Hodgkin lymphoma. J Clin Oncol 2017;35:2125-2132.

21 Bellmunt J, Bajorin DF: Pembrolizumab for advanced urothelial carcinoma. N Engl J Med 2017;376:2304

22 Muro K, Chung HC, Shankaran V, Geva R, Catenacci D, Gupta S, Eder JP, Golan T, Le DT, Burtness B, McRee AJ, Lin CC, Pathiraja K, Lunceford J, Emancipator K, Juco J, Koshiji M, Bang YJ: Pembrolizumab for patients with PD-L1-positive advanced gastric cancer (KEYNOTE-012): a multicentre, open-label, phase $1 \mathrm{~b}$ trial. Lancet Oncol 2016

23 Smyth EC, Cunningham D: Encouraging results for PD-1 inhibition in gastric cancer. Lancet Oncol 2016; 17:682-683.

24 Bang Y-J, Muro K, Fuchs CS, Golan T, Geva R, Hara H, Jalal SI, Borg C, Doi T, Wainberg ZA: KEYNOTE-059 cohort 2: safety and efficacy of pembrolizumab (pembro) plus 5-fluorouracil (5-FU) and cisplatin for first-line (1L) treatment of advanced gastric cancer. J Clin Oncol 2017;35:4012.

25 Catenacci Daniel V, Wainberg Z, Fuchs Charles S, Garrido M, Bang Y-J, Muro K, Savage M, Wang J, Koshiji M, Dalal Rita P: LBA-009 KEYNOTE-059 cohort 3: safety and efficacy of pembrolizumab monotherapy for first-line treatment of patients (pts) with PD-L1positive advanced gastric/gastroesophageal (G/GEJ) cancer. Ann Oncol 2017;28(suppl3):mdx302.008.

26 Fuchs CS, Doi T, Jang RW-J, Muro K, Satoh T, Machado M, Sun W, Jalal SI, Shah MA, Metges J-P: KEYNOTE-059 cohort 1: efficacy and safety of pembrolizumab (pembro) monotherapy in patients with previously treated advanced gastric cancer. J Clin Oncol 2017;35(suppl15):4003.

27 Wainberg Z, Jalal S, Muro K, Yoon H, Garrido M, Golan T, Catenacci D, Geva R, Ku G, Bleeker J: LBA28_PR KEYNOTE-059 update: efficacy and safety of pembrolizumab alone or in combination with chemotherapy in patients with advanced gastric or gastroesophageal (G/GEJ) cancer. Ann Oncol 2017;28 (suppl5): $\mathrm{mdx} 440.020$.

28 Tabernero J, Powderly JD, Hamid O, Gordon MS, Fisher GA, Braiteh FS, Garbo LE, Fine GD, Kowanetz M, McCall B, Shen X, Chen DS, Kohrt HE: Clinical activity, safety, and biomarkers of MPDL3280A, an engineered PD-L1 antibody in patients with locally advanced or metastatic CRC, gastric cancer (GC), SCCHN, or other tumors. J Clin Oncol 2013;31:3622-3622.

29 Chung HC, Arkenau H-T, Wyrwicz L, Oh D-Y, Lee K-W, Infante JR, Lee SS, Lee J, Keilholz U, Mita AC, Plummer ER, Kemeny M, Melichar B, Smith DM, Chin KM, von Heydebreck A, Cuillerot J-M, Kang Y-K, Safran H: Avelumab (MSB0010718C; anti-PDL1) in patients with advanced gastric or gastroesophageal junction cancer from JAVELIN solid tumor phase Ib trial: analysis of safety and clinical activity. J Clin Oncol 2016;34(suppl15):4009.
30 Moehler MH, Cho JY, Kim YH, Kim JW, Di Bartolomeo M, Ajani JA, Yamaguchi K, Balogh A, KongSanchez MT, Bang Y-J: A randomized, open-label, two-arm phase II trial comparing the efficacy of sequential ipilimumab (ipi) versus best supportive care (BSC) following first-line (1L) chemotherapy in patients with unresectable, locally advanced/metastatic (A/M) gastric or gastro-esophageal junction (G/GEJ) cancer. J Clin Oncol 2016;34(suppl15):4011.

31 Nishina T, Shitara K, Iwasa S: Safety, PD-L1 expression, and clinical activity of avelumab (MSB0010718C), an anti-PD-L1 antibody, in Japanese patients with advanced gastric or gastroesophageal junction cancer. J Clin Oncol 2016;34(suppl4):168.

32 Bang Y-J, Wyrwicz L, Park YI, Ryu M-H, Muntean A, Gomez-Martin C, Guimbaud R, Ciardiello F, Boku N, Van Cutsem E: Avelumab (MSB0010718C; anti-PDL1)+ best supportive care (BSC) vs BSC \pm chemotherapy as third-line treatment for patients with unresectable, recurrent, or metastatic gastric cancer: The phase 3 JAVELIN Gastric 300 trial. J Clin Oncol 2016;34 (suppl15):TPS4135.

33 Segal NH, Antonia SJ, Brahmer JR, Maio M, BlakeHaskins A, Li X, Vasselli J, Ibrahim RA, Lutzky J, Khleif S: Preliminary data from a multi-arm expansion study of MEDI4736, an anti-PD-L1 antibody. J Clin Oncol 2014;32(suppl15):3002.

34 Tabernero J, Powderly JD, Hamid O, Gordon MS Fisher GA, Braiteh FS, Garbo LE, Fine GD, Kowanetz M, McCall B: Clinical activity, safety, and biomarkers of MPDL3280A, an engineered PD-L1 antibody in patients with locally advanced or metastatic CRC, gastric cancer (GC), SCCHN, or other tumors. J Clin Oncol 2013;31(suppl15):3622.

35 Bang YJ, Cho JY, Kim YH, Kim JW, Di Bartolomeo M, Ajani JA, Yamaguchi K, Balogh A, Sanchez T, Moehler M: Efficacy of sequential ipilimumab monotherapy versus best supportive care for unresectable locally advanced/metastatic gastric or gastroesophageal junction cancer. Clin Cancer Res 2017;23:5671-5678.

36 Janjigian YY, Ott PA, Calvo E, Kim JW, Ascierto PA, Sharma P, Peltola KJ, Jaeger D, Evans TRJ, Braud FGD, Chau I, Tschaika M, Harbison CT, Cai W, Bendell JC, Le DT: Nivolumab \pm ipilimumab in pts with advanced (adv)/metastatic chemotherapy-refractory (CTx-R) gastric $(\mathrm{G})$, esophageal (E), or gastroesophageal junction (GEJ) cancer: CheckMate 032 study. J Clin Oncol 2017;35(suppl15):4014-4014

37 Bang Y-J, Muro K, Fuchs CS, Golan T, Geva R, Hara H, Jalal SI, Borg C, Doi T, Wainberg ZA: KEYNOTE-059 cohort 2: safety and efficacy of pembrolizumab (pembro) plus 5-fluorouracil (5-FU) and cisplatin for first-line (1L) treatment of advanced gastric cancer. J Clin Oncol 2017;35(suppl15):4012.

38 Yuan J, Zhou J, Dong Z, Tandon S, Kuk D, Panageas KS, Wong P, Wu X, Naidoo J, Page DB, Wolchok JD, Hodi FS: Pretreatment serum VEGF is associated with clinical response and overall survival in advanced melanoma patients treated with ipilimumab. Cancer Immunol Res 2014;2:127-132.

39 Oyama T, Ran S, Ishida T, Nadaf S, Kerr L, Carbone DP, Gabrilovich DI: Vascular endothelial growth factor affects dendritic cell maturation through the inhibition of nuclear factor- $\kappa \mathrm{B}$ activation in hemopoietic progenitor cells. J Immunol 1998;160:1224-1232.

40 Chau I, Penel, N, Arkenau, HT: Safety and antitumor activity of ramucirumab plus pembrolizumab in treatment naïve advanced gastric or gastroesophagealo junction (G/GEJ) adenocarcinoma: preliminary results from a multi-disease phase I study (JVDF). J Clin Oncol 2018;36(suppl4):101. 
41 Bang Y, Golan, T, Lin, CC, Kang YK, Wainberg ZA, Wasserstrom H, Jin J, Mi G, McNeely S, Laing N, Williams Goff L, Fu S: Interim safety and clinical activity in patients (pts) with locally advanced and unresectable or metastatic gastric or gastroesophageal junction (G/GEJ) adenocarcinoma from a multicohort phase I study of ramucirumab plus durvalumab. J Clin Oncol 2018;36(suppl4):92.

42 Cancer Genome Atlas Research Network: Comprehensive molecular characterization of gastric adenocarcinoma. Nature 2014;513:202-209.

43 Derks S, Liao X, Chiaravalli AM, Xu X, Camargo MC, Solcia E, Sessa F, Fleitas T, Freeman GJ, Rodig SJ, Rabkin CS, Bass AJ: Abundant PD-L1 expression in Epstein-Barr Virus-infected gastric cancers. Oncotarget 2016;7:32925-32932.
44 Cho J, Lee J, Bang H, Kim ST, Park SH, An JY, Choi MG, Lee JH, Sohn TS, Bae JM, Kang WK, Kim S, Kim KM: Programmed cell death-ligand 1 expression predicts survival in patients with gastric carcinoma with microsatellite instability. Oncotarget 2017;8:1332013328.

45 Chiaravalli AM, Feltri M, Bertolini V, Bagnoli E, Furlan D, Cerutti R, Novario R, Capella C: Intratumour T cells, their activation status and survival in gastric carcinomas characterised for microsatellite instability and Epstein-Barr virus infection. Virchows Arch 2006;448: 344-353.

46 Sohn BH, Hwang JE, Jang HJ, Lee HS, Oh SC, Shim JJ, Lee KW, Kim EH, Yim SY, Lee SH, Cheong JH, Jeong W, Cho JY, Kim J, Chae J, Lee J, Kang WK, Kim S, Noh SH, Ajani JA, Lee JS: Clinical significance of four molecular subtypes of gastric cancer identified by The Cancer Genome Atlas Project. Clin Cancer Res 2017; 23:4441-4449.
47 Davoli T, Uno H, Wooten EC, Elledge SJ: Tumor aneuploidy correlates with markers of immune evasion and with reduced response to immunotherapy. Science 2017;355:261.

48 Secrier M, Li X, de Silva N, Eldridge MD, Contino G, Bornschein J, MacRae S, Grehan N, O'Donovan M, Miremadi A, Yang TP, Bower L, Chettouh H, Crawte J, Galeano-Dalmau N, Grabowska A, Saunders J, Underwood T, Waddell N, Barbour AP, Nutzinger B, Achilleos A, Edwards PA, Lynch AG, Tavare S, Fitzgerald RC: Mutational signatures in esophageal adenocarcinoma define etiologically distinct subgroups with therapeutic relevance. Nat Genet 2016;48:1131-1141. 